\title{
Psychometric Properties of the Chinese version of the 20 -item Prosopagnosia Index (PI20)
}

\author{
Wen Sun ${ }^{1,2}$, Yuzheng Wang ${ }^{1,2}$, Jinyan Wang ${ }^{1,2 *}$, and Fei Luo ${ }^{1,2}$ \\ ${ }^{1}$ CAS Key Laboratory of Mental Health, Institute of Psychology, Chinese Academy of Sciences, Beijing, P.R. China.; \\ ${ }^{2}$ Department of Psychology, University of Chinese Academy of Sciences, Beijing, P.R. China;
}

\begin{abstract}
[Abstract] Prosopagnosia, also known as facial blindness, has attracted wide attention in the social field in recent years. However, it mainly depends on the self-report of the chief complaint in the actual diagnosis. In addition, there is a lack of standardized measurement basis, which brings a lot of inconvenience to the patients themselves and follow-up researchers. In this study, we tested the reliability and validity of the Chinese version of 20 - item prosopagnosia index (PI-20). 647 young people aged 18-45 filled in PI-20, the adult autistic quotient scale, the empathy quotient questionnaire, and completed the retest one month later. Results showed that the PI-20 has good internal consistency reliability, test-retest reliability, and has good validity. In addition, confirmatory factor analysis shows that the model can well fit the data of Chinese population and can be used for large-scale measurement. The Chinese version PI-20 is a reliable measurement for prosopagnosia.
\end{abstract}

\section{Introduction}

Individuals with prosopagnosia are unable to recognize acquaintances and their own faces while their intelligence, social function and other aspects of visual processing (such as object recognition) are normal. Bodamer, a famous German neurologist and psychiatrist, first proposed the term -- prosopagnosia in 1947[1]. The researchers gradually differentiated the term into acquired prosopagnosia (AP) caused by brain injury and relative developmental prosopagnosia (DP) according to the different conditions of the patients with the deepening of the study and the increase of patient cases. The symptoms of face recognition disorder in patients with AP were significantly different before and after the onset of the disease, and the ability of face recognition was lost only after the disease. However, some DP patients have symptoms that occur at an early stage of development, and never have normal facial recognition. In addition, they do not realize that they are different from others [12].

Prosopagnosia, or so-called "face blindness" is attracting the attention of the scholars. In addition, people's understanding of this concept often becomes a reason to make fun of themselves or avoid social interaction. However, the real difficulty in face recognition can bring great social and psychological troubles to patients. For example, some patient case reports show that when they add the more complex social scenes of people such as schools / workplaces, they will bring social frustration because of ignoring acquaintances or recognizing the wrong person. It will lead to strong social anxiety in the long run and lead to difficulties in the development of social relations. In addition, even mention these experiences in some cases, it can affect their academic development or career choices [7]. For people who claim to be face-blind in daily life, it is obvious that most of them have not experienced brain trauma. As a consequence, the exact diagnosis is of great significance to the social life of those patients with real prosopagnosia. On the one hand, they can find the real reasons for their impolite behavior and obtain understanding. On the other hand, they can be targeted treatment to improve the ability of face recognition.

The diagnosis of prosopagnosia needs to be combined with a variety of methods at present. It often starting from patients' complaints about face recognition in their daily life [4]. The main research methods are conducted through self-reporting or structured / semi-structured interviews, such as asking potential patients about the experience of face recognition in daily life [13]. However, self-reporting is limited by individual cognition, and the cost of interview method is high. In addition, information recording is troublesome, and the processing results are difficult to carry out quantitative analysis. The lack of standardized diagnosis leads to difficulties in the comparison of cases, which limits the follow-up research. As a consequence, some questionnaires with good psychometric properties have been gradually developed, such as the questionnaire of 15 items developed by Kennerknecht et al. and the 20 - item prosopagnosia index (PI-20) developed by Shah et al and so on. PI-20 has been proved to have good structure, reliability and

* Jinyan Wang: wangjy@psych.ac.cn 
validity in the study of Portuguese population [14]. Unfortunately, no Chinese version has been published yet. Based on this, we intend to introduce prosopagnosia index (PI-20) into China to help potential patients better identify and judge their own condition. In addition, we are committed to providing a more unified and reliable measurement tool for follow-up researchers in the study of prosopagnosia.

\section{Objects and methods}

\subsection{Materials}

The 20 - item prosopagnosia index, published by Shah et al in 2015, consists of 20 short descriptions of daily conditions. The subjects were asked to judge to what extent the situation in the item statement was consistent with their face recognition ability and experience in daily life. In addition, the questionnaire uses a 5-point scale, with options ranging from "very disagree" to "very agree". Fifteen of the presentation options were positive scores, that is, 5 points for "very much agree", 1 point for very disagreement, and 5 reverse scores for the other 5 items. The final total score varies from 20 to 100 [6].

The Adult Autistic Quotient scale (AQ), developed by Professor Baron-Cohen and his team, is used to measure autistic traits in normal people. There are 50 questions, which can be divided into five subscales: social skills, attention transfer, attention to detail, communication skills, and imagination. The total score of the questionnaire ranges from 0 to 50 . The higher the score, the more obvious the autistic trait is. 24 positive questions and 26 reverse questions were randomly arranged. The subjects scored 1 when they rated the positive question as "a little agree" or "completely agreed", and 0 when they rated themselves as "totally disagree" or "a little disagree". The scoring rule of the reverse question was on the contrary [11].

The empathy Quotient questionnaire (EQ) is developed for diagnostic purposes, especially for measuring the empathy ability of autistic patients. It has good reliability and validity in measuring the empathy ability of Asperger's disease, autistic people and healthy people. The questionnaire is a self-rating scale with a total of 60 questions. Each question has 4 choices according to the degree of compliance. "Very much agree" scored 2 points, "a little agree" scored 1, "a little disagreed" and "very disagreed" scored 0. Some topics were scored backward, that is, "very disagreed" scored 2 points; another 20 questions were filled items, and the results of the options had no effect on the total score. The final total score is $0-80$ points range [15].

\subsection{Method}

The Chinese version of the questionnaire was translated into Chinese by the researchers and the first draft was finalized after discussion by the research group. For those words in doubt, we confirm them directly with the original author. In addition, it was translated back into English to make sure that the Chinese meaning is similar to the English meaning according to the revised procedure of the cross-cultural scale. Finally, the content of the Chinese version of prosopagnosia index questionnaire (PI-20) was determined. The method of data collection is online questionnaire test. The statistical analysis of the data was mainly done by SPSS25.0, and the confirmatory factor analysis (CFA) was completed by SPSSAU22.0.

\subsection{Subjects}

647 young people (female, $71.7 \%$ ) between the ages of 18 and 45 filled in the prosopagnosia index (PI-20) questionnaire, the adult autistic quotient scale (AQ) and the empathy quotient questionnaire (EQ) online twice with an interval of a month. The mean age was 22.11 years old, and the standard deviation was 3.41. People aged between 18 and 25 accounted for $88.6 \%$ of the total.

\section{Results}

\subsection{Project Analysis}

In the 20 - item prosopagnosia index (PI-20) questionnaire, the scores of 20, except for item 3 were positively correlated with the total score. The minimum and maximum correlation coefficient was 0.43 and 0.844 , respectively, and all had significant significance (Table $1)$.

Table 1 Correlation coefficient between total score and project score

\begin{tabular}{cccc}
\hline Item number & $\begin{array}{c}\text { Correlation } \\
\text { coefficient }\end{array}$ & Item number & $\begin{array}{c}\text { Correlation } \\
\text { coefficient }\end{array}$ \\
\hline 1 & $0.830^{* *}$ & 11 & $0.661^{* *}$ \\
2 & $0.844^{* *}$ & 12 & $0.805^{* *}$ \\
3 & -0.036 & 13 & $0.430^{* *}$ \\
4 & $0.780^{* *}$ & 14 & $0.675^{* *}$ \\
5 & $0.712^{* *}$ & 15 & $0.777^{* *}$ \\
6 & $0.773^{* *}$ & 16 & $0.676^{* *}$ \\
7 & $0.794^{* *}$ & 17 & $0.607^{* *}$ \\
8 & $0.561^{* *}$ & 18 & $0.532^{* *}$ \\
9 & $0.605^{* *}$ & 19 & $0.533^{* *}$ \\
10 & $0.631^{* *}$ & 20 & $0.674^{* *}$ \\
\hline$* p<0.05, * * p<0.01$ & &
\end{tabular}

\subsection{Confirmatory factor analysis}

We conducted a confirmatory factor analysis of the data by single-factor model. The purpose of this study is to test whether the 20 - item prosopagnosia index (PI-20) 
questionnaire is suitable for young people in China. The indicators are shown in table 2.

Table 2 Model fitting index

\begin{tabular}{lccccccccc}
\hline Index & $\chi^{2}$ & df & $\chi^{2} / d f$ & GFI & RMSEA & CFI & NFI & TLI & AGFI \\
\hline criterion & - & - & $<3$ & $>0.9$ & $<0.10$ & $>0.9$ & $>0.9$ & $>0.9$ & $>0.9$ \\
Value & 427.06 & 159.00 & 2.69 & 0.93 & 0.05 & 0.96 & 0.94 & 0.95 & 0.91 \\
\hline
\end{tabular}

Note: $\chi{ }^{2}$ is the chi-square value; $\mathrm{df}$ is the degree of freedom; GFI is the goodness-of-fit index; RMSEA is the root mean square of the approximate error; CFI is the comparative fitting index; NFI is the standard fitting index; TLI is the Tucker-Lewis index; AGFI is the goodness-of-fit index.

As shown in Table 2, the fitting indexes of the model are good. It indicates that the model of PI-20 questionnaire proposed by Shah et al has a certain explanatory power, and also fits the data obtained by Chinese youth groups well.

Table 3 Table of factor load factors

\begin{tabular}{ccccccc}
\hline Factor & $\begin{array}{c}\text { Analysis } \\
\text { item }\end{array}$ & Coef & Std. Error & $\mathrm{z}$ & $\mathrm{p}$ & $\begin{array}{c}\text { Std. } \\
\text { Estimate }\end{array}$ \\
\hline Factor 1 & 1 & 1 & - & - & - & 0.811 \\
& 2 & 0.992 & 0.025 & 39.161 & 0 & 0.826 \\
& 3 & -0.059 & 0.035 & -1.698 & 0.089 & -0.069 \\
& 4 & 0.893 & 0.039 & 22.813 & 0 & 0.784 \\
5 & 0.659 & 0.033 & 20.069 & 0 & 0.713 \\
6 & 0.837 & 0.038 & 22.145 & 0 & 0.767 \\
& 7 & 0.943 & 0.041 & 23.252 & 0 & 0.796 \\
& $8^{*}$ & 0.513 & 0.041 & 12.533 & 0 & 0.481 \\
$9^{*}$ & 0.582 & 0.041 & 14.148 & 0 & 0.535 \\
10 & 0.578 & 0.034 & 17.073 & 0 & 0.627 \\
11 & 0.661 & 0.038 & 17.226 & 0 & 0.645 \\
12 & 0.911 & 0.038 & 23.736 & 0 & 0.807 \\
$13^{*}$ & 0.358 & 0.038 & 9.445 & 0 & 0.371 \\
14 & 0.757 & 0.041 & 18.249 & 0 & 0.661 \\
15 & 0.799 & 0.035 & 22.881 & 0 & 0.787 \\
16 & 0.795 & 0.045 & 17.49 & 0 & 0.639 \\
$17 *$ & 0.573 & 0.041 & 14.047 & 0 & 0.532 \\
18 & 0.525 & 0.041 & 12.959 & 0 & 0.495 \\
$19^{*}$ & 0.492 & 0.042 & 11.824 & 0 & 0.456 \\
20 & 0.667 & 0.037 & 18.229 & 0 & 0.661 \\
\hline
\end{tabular}

Note: $*$ is the reverse of several questions

As shown in Table 3, the factor loading value shows the correlation between the factor (latent variable) and the analysis term (explicit variable). Except for item 3 and item 13 in the questionnaire, the other items have a strong correlation.

\subsection{Reliability test}

Cronbach questionnaire was used to test the internal consistency of the scale according to the collected data $(\mathrm{N}=647)$. As a result, it showed that the questionnaire has an acceptable internal consistency coefficient. The overall $\alpha$ of 20 items was 0.929 .

The test-retest reliability of 647 subjects after an interval of one month was 0.884 . It indicated that the consistency of the measured scores before and after the test was high and the stability was good.

\subsection{Validity test}

The correlation between PI-20 and AQ questionnaire, PI-20 and EQ questionnaire was analyzed to see if the total score of the scale conformed to the theoretical hypothesis in order to measure the correlation validity. The results were shown in the table 4 .

Table 4 PI-20 is related to other scales

\begin{tabular}{ccccc}
\hline & $\begin{array}{c}\text { Preliminary } \\
\text { measurement } \\
\text { AQ }\end{array}$ & $\begin{array}{c}\text { Retest } \\
\text { AQ }\end{array}$ & $\begin{array}{c}\text { Preliminary } \\
\text { measurement } \\
\text { EQ }\end{array}$ & $\begin{array}{c}\text { Retest } \\
\text { EQ }\end{array}$ \\
\hline $\begin{array}{c}\text { Preliminary } \\
\text { measurement } \\
\text { PI }\end{array}$ & $0.304 * *$ & $0.314 * *$ & $-0.301 * *$ & $-0.285^{* *}$ \\
Retest PI & $0.277^{* *}$ & $0.322^{* *}$ & $-0.275^{* *}$ & $-0.306^{* *}$ \\
\hline$* p<0.05, * * p<0.01$ & & &
\end{tabular}

As shown in Table 4, the total score of PI-20 was positively correlated with the total score of AQ and negatively correlated with the total score of EQ. The results were consistent with the research hypothesis.

\section{Discussion}

The Chinese version of the PI-20 has good internal consistency reliability, test-retest reliability, and has good validity. In addition, confirmatory factor analysis shows that the model can well fit the data of Chinese population and can be used for large-scale measurement. The Chinese version PI-20 is a reliable measurement for prosopagnosia.

The purpose of the 20 - item prosopagnosia index (PI-20) is to help potential patients understand their actual situation and provide a reliable measurement tool for follow-up research on prosopagnosia. The results of confirmatory factor analysis for PI-20 in this study show that the overall fit of the questionnaire is good. Only the correlation coefficient of the item 3 is opposite to the prediction and is not significant. In addition, the standard load coefficient of the item 13 is slightly lower, which is still room for further optimization. Now reviewing the content of the question: item 3; "I found it significantly easier to recognize people with unique facial features," even people with normal facial recognition might still think they were inclined to agree. Perhaps the reverse question "it is more difficult to identify people without unique facial features" can more easily reflect the true intention of the item. The lower load coefficient in item 13 may mean that even in groups with certain defects in face recognition. In addition, there are still fewer people who cannot recognize their own faces. Considering the cultural differences between the East and the West, and we still hope to retain the possibility of further verifying these two questions in future studies. As a consequence, these types of questions have not been deleted in this revision of the questionnaire in order to ensure the integrity of the questionnaire as a whole.

The reliability test results show that the scale is stable and reliable. Its internal consistency is higher than 0.7 , 
indicating that each project has reached a good level of internal homogeneity. The test-retest reliability of the total score of the scale was 0.884 in the test-retest study of the same group with an interval of one month. It indicates that the score of the scale had good internal consistency and cross-time stability.

There was a significant correlation between the 20 item prosopagnosia index (PI-20) questionnaire and the adult autistic quotient questionnaire (AQ) and the empathy scale (EQ). It showed that this scale has a certain correlation validity. Autistic patients generally have obstacles in face naming, recognition, coding and other tasks from previous studies. It may be due to the formation of their unique eye movement gaze pattern, or because of the same neural mechanism behind both [18-20]. Based on the theoretical hypothesis, we verified the correlation between the PI-20 questionnaire and the AQ questionnaire. As a result, it showed a significant positive correlation. In the meanwhile, empathy deficiency is also a typical characteristic of autistic patients. The face recognition ability which is positively correlated with AQ should be negatively correlated with empathy quotient EQ. In fact, this result can also be shown in the revised analysis of this scale.

\section{Conclusion}

In general, the Chinese version of the 20 - item prosopagnosia index (PI-20) questionnaire has good reliability and criterion validity in Chinese youth groups. However, the performance of individual items (item 3, 13) needs to be further analyzed and optimized. In addition, the structure of the scale also needs to be further explored. Besides, the data collected from this revision work are relatively high in women and more concentrated in 18-25 years old. As a consequence, male population data and other age data can be supplemented later in order to more comprehensively reflect the reliability and validity of the 20 - item prosopagnosia index (PI-20) questionnaire in the Chinese population.

\section{Compliance with Ethical Standards}

Conflict of Interest On behalf of all authors, the corresponding author states that there is no conflict of interest.

Ethical Approval All procedures performed in studies involving human participants were in accordance with the ethical standards of the Institute of Psychology and with the 1964 Helsinki declaration and its later amendments or comparable ethical standards. No adverse events were reported in this study.

Informed Consent Informed consent was obtained from all individual participants.

\section{Acknowledgements}

This work was supported by the NNSF (National Natural Science Foundation of China) under Grant (31970926) to Fei Luo; the NNSF under grant (31271092) to Jinyan
Wang; the NNSF grant (31671140) to Ning Wang; the Scientific Foundation of Institute of Psychology, Chinese Academy of Sciences (Y9CX441005) to Yuzheng Wang; grants from CAS Key Laboratory of Mental Health, Institute of Psychology (KLMH 2014G01, KLMH2016K02) and a grant from the initiation fund of the CAS/SAFEA International Partnership Program for Creative Research Teams (Y2CX131003).

\section{References}

1. Corrow S L, Dalrymple $\mathrm{K}$ A, Barton J J.Prosopagnosia: current perspectives[J].Eye Brain,2016, 8: 165-175.

2. Dalrymple K A, Palermo R.Guidelines for studying developmental prosopagnosia in adults and children[J].Wiley Interdisciplinary ReviewsCognitive Science,2016, 7 (1): 73-87.

3. Fine D R.A life with prosopagnosia[J].Cognitive Neuropsychology,2012, 29 (5-6): 354-359.

4. Palermo R, Rossion B, Rhodes G, et al.Do people have insight into their face recognition abilities?[J].Quarterly journal of experimental psychology (2006),2017, 70 (2): 218-233.

5. Kennerknecht I, Kennerknecht I, Plümpe N, et al.Hereditary prosopagnosia (HPA): the first report outside the Caucasian population[J].Journal of human genetics,2007, 52 (3): 230-236.

6. Shah P, Gaule A, Sowden S, et al.The 20-item prosopagnosia index (PI20): a self-report instrument for identifying developmental prosopagnosia[J].Royal Society Open Science, 2015, 2

7. Yardley L, Mcdermott L, Pisarski S, et al.Psychosocial consequences of developmental prosopagnosia: a problem of recognition[J].J Psychosom Res,2008, 65 (5): 445-51.

8. Gruter T, Gruter M, Carbon C C.Neural and genetic foundations of face recognition and prosopagnosia[J].Journal of Neuropsychology, 2008, 2: 79-97.

9. Mcconachie $\mathrm{H} \quad \mathrm{R}$, Mcconachie $\mathrm{H}$ R.Developmental Prosopagnosia. A Single Case Report[J]. Cortex,1976, 12 (1): 76-82.

10. Shah P, Gaule A, Sowden S, et al.The 20-item prosopagnosia index (PI20): a self-report instrument for identifying developmental prosopagnosia[J].Royal Society Open Science, 2015, 2 (6).

11. Baron-Cohen S, Wheelwright S, Skinner R, et al.The autism-spectrum quotient (AQ): Evidence from Asperger Syndrome/high-functioning autism, males and females, scientists and mathematicians (vol 31, pg 5, 2001)[J].Journal of Autism and Developmental Disorders,2001, 31 (6): 603-603.

12. Arizpe J M, Saad E, Douglas A O, et 
al.Self-reported face recognition is highly valid, but alone is not highly discriminative of prosopagnosia-level performance on objective assessments[J].Behavior research methods,2019, 51 (3): 1102-1116.

13. Zhao Y F, Li J G, Liu X Q, et al.Altered spontaneous neural activity in the occipital face area reflects behavioral deficits in developmental prosopagnosia[J].Neuropsychologia,2016, 89 : 344-355.

14. Ventura P, Livingston L A, Shah P.Adults have moderate-to-good insight into their face recognition ability: Further validation of the 20-item Prosopagnosia Index in a Portuguese sample[J].Quarterly Journal of Experimental Psychology,2018, 71 (12): 2677-2679.

15. Simon Baron-Cohen, Sally Wheelwright.The empathy quotient: an investigation of adults with Asperger syndrome or high functioning autism, and normal sex differences. [J].Autism Dev Disord 2004,34(2):163-75.

16. Joseph M. Arizpe Elyana Saad Ayooluwa O. Douglas Laura Germine Jeremy B. Wilmer Joseph M. DeGutis.The relation between face-emotion recognition and social function in adolescents with autism spectrum disorders. A case control study.Behavior Research Methods, 2019, 51(3):1102-1116.

17. E. Loth,L. Garrido,J. Ahmad,E. Watson.A. Duff,B. Duchaine.Facial expression recognition as a candidate marker for autism spectrum disorder: how frequent and severe are deficits?Molecular Autism (2018) 9:7

18. Hanna B. Cygan,Hanna Okuniewska,Katarzyna Jednoróg,Artur Marchewka,Marek Wypych,Anna Nowicka.Face processing in a case of high functioning autism with developmental prosopagnosia.Acta Neurobiol Exp (Wars), 2018; 78(2):114-131

19. Ilaria Minio-Paluello, Giuseppina Porciello, Alvaro Pascual-Leone, Simon Baron-Cohen. Face individual identity recognition: a potential endophenotype in autism.Molecular Autism (2020) 11:81

20. L J Carver, G Dawson.Development and neural bases of face recognition in autism.Mol Psychiatry. 2002;7 Suppl 2:S18-20. 